\title{
The role of communication in cancer management
}

\author{
Kofi Adesi Kyei ${ }^{1, ~ *, ~ W i l l i a m ~ K w a d w o ~ A n t w i ~}{ }^{1}$, Michael Osei ${ }^{2}$, Lawrence Arthur ${ }^{1}$, \\ James Newlife Kwadzo Gawugah ${ }^{1}$ \\ ${ }^{1}$ Department of Radiography, School of Biomedical and Allied Health Sciences, University of Ghana, Korle-Bu, Accra, Ghana \\ ${ }^{2}$ National Centre for Radiotherapy and Nuclear Medicine, Korle-Bu, Accra, Ghana
}

Email address:

adesco41@hotmail.com (K. A. Kyei), kakyei@chs.ug.edu.gh (K. A. Kyei)

\section{To cite this article:}

Kofi Adesi Kyei, William Kwadwo Antwi, Michael Osei, Lawrence Arthur, James Newlife Kwadzo Gawugah. The Role of Communication in Cancer Management. Cancer Research Journal. Vol. 3, No. 1, 2015, pp. 1-5. doi: 10.11648/j.crj.20150301.11

\begin{abstract}
Background: Cancer management is increasingly being delivered within a multidisciplinary team (MDT) environment, involving several highly skilled professionals. There is therefore a good indication of the importance of good and effective communication skills within these collaborative care teams. Aim: The study examined the process of communication, information transfer and collaboration among the MDT in cancer management. Methods: A quantitative descriptive survey using a semi structured questionnaire was administered to Forty five (45) multidisciplinary health professionals. Data collected was analyzed using Statistical Package for Social Scientists (SPSS) version 18.0 and discussions were based on the responses from the participants. Results: More than half (54.5\%) of the respondents used verbal communication whiles $31.8 \%$ described telephone as the most difficult mode of communication for them. Lack of additional education in communication skills was identified among $75 \%$ of the respondents. There were $29.5 \%$ of the respondents who indicated that Staff especially the seniors show disrespect to the juniors. In all, $34 \%$ of the participants had no idea about whether protocols or guidelines in communication existed. Conclusion: There was a high level collaboration level among the MDT involved in the management of cancer. Most of these professionals however, had no further education in communication skills and several recommendations were on In-Service Trainings be organize for the various disciplines on regular basis for all the staff.
\end{abstract}

Keywords: Communication, Collaboration, Cancer Care, Professional, MDT

\section{Introduction}

The increasing cost of healthcare and rapid growth in knowledge has compelled healthcare professionals to share knowledge and skills. Such collaboration requires interaction, which has led to an increased focus on the subject of communication skills [1]. Communication derived from a Latin word "communis", is the activity of conveying or sharing information [2]. Multidisciplinary involvement from the early stages of cancer management ensures that a full therapeutic range of options are considered so that patients receive appropriate and timely treatment [3].

The Canadian Medical Protective Association (CMPA) [4] indicated that good patient care depends on good communication among the members of a team. Another study by Fox [5] pointed out that explicit communication strategies are needed to maintain quality in patient care. Another study [6] showed that 259 patients out of all patients undergoing Radiotherapy between 1997 and 2002 were wrongly done due to an inadequate/incorrect documentation of technical changes due to lack of communication. In view of this, The Royal College of Surgeons in England [7] explained that handing over should be a two-way process to provide and receive information, and gives an opportunity to ask questions. They indicated that clinical staff should make each other aware of relevant issues.

Evidence suggests that when health providers and patients speak different languages, achieving effective communication becomes immeasurably more challenging [8]. Lee [8] pointed out that language and cultural barriers in health communication is one of the many challenges facing health care in the United States and it affects the quality and accessibility of health care.

The World Health Organization (WHO) profile on Radiotherapy risk revealed that misinformation or errors in data transfer, constituted the majority of incidents in modern Radiotherapy services [9]. It was established between 1990-1991 and 1995-1999 in Japan that differences in the interpretations of a prescribed dose between Radiation Oncologists and Therapy Radiographers resulted in an 
overdose for 276 patients.

Lack of communication within the medical field may result in medical errors and this may lead to severe injury or unexpected patient death. Medical errors, especially those caused by a failure to communicate, are a pervasive problem in today's health care organizations [10]. The Joint Commission on Accreditation of Healthcare Organisation (JCAHO), admitted that lack of critical information, misinterpretation of information, unclear orders over the telephone, and overlooked changes in status were the outcomes of miscommunication among health professionals which in effects puts patients at risk [11]. The Institute of Medicine (IOM) report indicated that between 44,000 and 98,000 people die every year in the U.S.A hospitals as a result of medical errors attributed to lack of communication [12].

In Ghana, MDT approach is employed and patients go through a process which requires the services of different health professionals. All activities such as clinical and transfer of information between staff to staff and staff to patients are carried out through communication. Through communication, information transfer and collaboration activities go on smoothly. The nature of the treatment is such that any error or misinformation within the radiotherapy process can be fatal to the patient. Till date no major study has been done to evaluate the effectiveness of information transfer and communication at Oncology Unit in Ghana. The focus of this study was therefore to identify whether communication, information transfer and collaboration among the health professionals at the Unit were of optimum standard.

\section{Methodology}

A quantitative descriptive survey was employed to assess communication skills and transfer of information among the patients. The study was conducted in one of the Teaching Hospitals in Ghana. The target population for the study were health Professionals, who form part, share and transfer patient information among themselves as well as attends to the patient directly or indirectly in an Oncology Department. These workers included Oncologists, Radiotherapist, Medical physicists, Pharmacists, Oncology nurses and their assistants, Record keepers, Receptionists and Secretaries.

The target population comprised of forty-nine professionals who were in active post at the time of study. From the total population size of forty-nine with a confidence level of $95 \%$, a sample size of forty five (45) was generated. A non-probability purposive sampling method was therefore employed for distribution and collection of data from the health professionals needed for the study which were in all 45 .

A structured questionnaire made-up of closed and few open ended types of questions was used to collect data from respondents. The respondents were made to choose a possible best from a set of answers and also express their opinion where necessary without being influenced whiles the closed ended questions limited the respondents to the set of alternative and precise answers provided. It was in 4 sections; the first section covered the demographics of participants whiles the other three parts collected data on how information were transferred, mode of communication and team collaboration. Out of the 45 questionnaires distributed, 44 were completed and returned. Data from 44 respondents were analyzed using Statistical Package for Social Scientists (SPSS version 18.0) and Microsoft excel 2007. Tables and Graphs were generated in graphical presentations. All statistical tests were conducted as two-sided, and declared significant for $\mathrm{p}$-value $<0.05$.

Approval for the study was obtained from the research ethics committee of a higher education institution. The ethics approval was supported by written permission for the study to be conducted at the study site. All study participants gave informed consent prior to the commencement of the study.

\section{Results}

The response rate of this study was with female to male ratio of $1: 2$. In all, $52 \%$ of the sample had postgraduate level of education, $16 \%$ had first degree where as only $11 \%$ formed the certificate. All respondents spoke English, while $65 \%$ spoke Twi which was a local language, $14 \%$ could speak French though Ghana is not a French speaking country. Majority (79.5\%) of the respondents could speak at least three languages and lof the respondents spoke six languages.

More than half $(54.5 \%)$ of the respondents used verbal communication, $27.3 \%$ used documentation while only $6.8 \%$ used DICOM. $31.8 \%$ described telephone as the most difficult mode of communication for them and $27.3 \%$ reported on DICOM. Regarding patient management, $86 \%$ of the patients indicated the interactions with doctors, $79 \%$ with radiotherapist while $54 \%$ interacted with the pharmacists.

Table 1. Distribution of level of collaboration among health workers.

\begin{tabular}{lllll}
\hline Health Worker & $\begin{array}{l}\text { Very High } \\
(\%)\end{array}$ & $\begin{array}{l}\text { High } \\
(\%)\end{array}$ & Low (\%) & $\begin{array}{l}\text { Very low } \\
(\%)\end{array}$ \\
\hline Oncologist & 82 & 7 & 6 & 5 \\
Pharmacist & 74 & 17 & 6 & 3 \\
Medical Doctor & 71 & 14 & 10 & 5 \\
Radiotherapist & 76 & 13 & 7 & 4 \\
Medical & 77 & 16 & 4 & 3 \\
physicist & & 31 & 2 & 0 \\
Nurse & 67 & 18 & 29 & 15 \\
Engineers & 38 & 41 & 19 & 0 \\
Secretaries & 48 & 26 & 11 & 10 \\
Receptionist & 53 & 14 & 31 & 20 \\
Records & 35 & & & \\
\hline
\end{tabular}

Majority of the respondents (82\%) reported very high collaboration with Oncologist as well as the Radiotherapist $(76 \%)$.

Only $38.6 \%$ of respondents were sure that protocols and guideline for communication existed while $27.3 \%$ had no idea of its existence. Only $25 \%$ of respondents indicated that they had additional training in communications and information transfer. 
Table 2. Distributions for communication protocols and education.

\begin{tabular}{cccl}
\hline $\mathbf{R}$ & Response & Frequency & Percent $\mathbf{( \% )}$ \\
\hline Protocols for communication exist & & \\
Yes & 17 & 38.6 \\
No & 15 & 34.1 \\
No Idea & 12 & 27.3 \\
Total & 44 & 100 \\
Additional education/training in communication & \\
Yes & 11 & 25.0 \\
No & 33 & 75.0 \\
Total & 44 & 100 \\
\hline
\end{tabular}

Table 3. Distribution for information and language problem.

\begin{tabular}{lll}
\hline & Frequency & Percent (\%) \\
\hline Protocols for communication exist & & \\
Reject the information & 2 & 4.5 \\
Carry on task & 3 & 6.8 \\
Ask for clarification & 37 & 84.1 \\
Refer from similar information & 2 & 4.5 \\
Attending to Patient with language problem & \\
Sign language & 6 & 13.6 \\
Interpreter & 38 & 86.4 \\
Refer patient & 0 & 0 \\
Use experience & 0 & 0 \\
\hline
\end{tabular}

Almost all (84.1\%) of the respondents asked for clarification from source when they receive ambiguous information. $86.4 \%$ representing most of the staff uses the services of an interpreter when attending to a patient with language problem.

Table 4. Distribution for communication errors.

\begin{tabular}{lll}
\hline & Frequency & Percent (\%) \\
\hline Errors due to miscommunication & 30 & \\
Yes & 10 & 68.2 \\
No & 4 & 22.7 \\
No Idea & 10 & 9.1 \\
Unavailable measures to minimize errors & \\
Regular quality assurance & 8 & 22.7 \\
Clinical meeting & 2 & 18.2 \\
CPD meeting & 19 & 4.5 \\
In-service training & 5 & 43.2 \\
No measures & & 11.4 \\
\hline
\end{tabular}

More than half of the respondents $(68.2 \%)$ identified errors due to communication. $43.2 \%$ of the staff reported that the centre lacks in-service training as a measure of minimising communication error.

\section{Discussion}

The study revealed that all, $100 \%$ of the respondents speak the English language. Among over 100 languages and dialects in the country only Ga, Twi and Ewe were the predominant local languages spoken by the respondents.

\section{Collaboration among the Multidisciplinary Team}

The findings of the study revealed that apart from the Doctors and the Therapy Radiographers whose role brings them in contact with most of the other workers, almost all 93.2\% work with oncologists and nurses regarding patient management while only $54 \%$ with pharmacists (Table 1 ). The workers coordinate more with some professionals than others when it comes to patient management therefore it was not surprising that majority of the respondents $(82 \%)$ reported very high collaboration with Oncologist. It is imperative that there should be respect among the professionals [13] in spite of hierarchical difference in the nature of their professions to achieve a collaborative working environment.

\section{Communication and Information Transfer}

In the results (Table 2 ) only $38.6 \%$ of the respondents were aware of the existence of protocols and guidelines in communication at the centre. The rest had no idea neither did they believe that there were protocols and guidelines in communication. This result could be disturbing since strong recommendations have been made for trainings in communication skills [14]. Moreover good communication is also a component of good clinical care [15]. Moreover, 54.5\% of the respondents communicate verbally or via oral instructions.

Literatures $[16,17]$ have however shown that the use of DICOM, even though has become a predominant standard for the communication of medical images in imaging departments, understanding of its operations is limited. Telephone communication is more accessible in the centre since each office is provided with a telephone connected to an intercom system; however some of the respondents reported it as the most difficult medium for communication. This could probably be due to many factors such as; hearing ability of the receiver, authenticity of the information being delivered and lengthy messages which can easily be forgotten [18].

A significant $27.3 \%$ reported on DICOM as also a difficult medium which is obvious due to its complexity $[16,17]$ while half $(50 \%)$ of the respondents found documentation as the easiest and effective medium in receiving information.

More than half of the respondents received clear and precise information from colleagues. Meanwhile a significant $27.3 \%$ reported that though they receive clear information, it is ambiguous. However, majority (84.1\%) indicated that they would request for clarification from the source of the information for accuracy (Table 3) when they detect ambiguity in patient information. A recommendation by WHO [9] for safety practices stresses on error reporting and quality improvement in radiotherapy treatment which may result in fatal situations and are irreversible. Again, during 
staff-patient communication, even though $13.6 \%$ indicated using sign language to attend to patients with language problem, a greater number of the respondents $(86 \%)$ were of the view that interpreters would be required especially patients from neighbouring countries such as Ivory Coast, Togo, Benin, Burkina Faso and other foreign countries (Table $3)$. The study indicated that majority $(68.2 \%)$ had detected technical or clinical errors due to miscommunication (Table 4) and as part of the measures in minimising these inaccuracies, most (43\%) of the respondents is of the view that the staff will need an in-service training. Butow [15] suggested that training in communication skills is essential, especially in cancer care. Again, a significant $22.7 \%$ of the respondents were of the view that the department regular quality assurance will be needed which is actually recommended as one of the safety measures to ensure quality radiotherapy delivery [9].

Almost 39\% reported on professional status as a hindrance to an effective communication while $15.9 \%$ indicated that position occupied by the staff. This was not surprising as O'Daniel \& Rosenstein [10] have mentioned these barriers in a list of some common barriers in inter-professional communication and collaboration. The sparingly distributed result may mean that the effect of these communication barriers recorded could not be severe within the department. However, in ensuring effective communication, respondents suggested on in-service training, quality assurance, provision of protocols on effective communication and frequent training in effective communication.

\section{Conclusion}

The study presents a helpful insight into the communication processes and collaborative measures that enhances effective patient care in cancer management at the study site. Documentation and oral or verbal instructions were the predominant media through which the professionals communicated and transferred information. The study also indicated that professionals, who attend directly to patients that had language problem. The absence or unavailability of protocols, guidelines and in-service trainings for staff members to an effective communication was however a challenge. Hence, developmental and evaluation of interventions based on these findings would be needed to improve the quality and effective mode of communication, information transfer as well as collaboration among the MDT in cancer management.

Some recommendations made to help the current practice were that protocols and guidelines in communication, especially in patient information transfer and staff handover must be outlined and instituted at all levels in the department for effective hand over and communication in cancer management. Also, the roles and responsibilities of professionals should be made known to other professionals to encourage co-ordination and collaboration among the multidisciplinary team. Finally, in-service training and continuous professional development (CPD) programs must be organized on regular basis to educate staff on structured communication tools to provide effective and better clarity in inter-professional communication.

\section{References}

[1] Skeff, K.M. \& Mutha S. (1998). Role models - Guiding the future of medicine. The New England Journal of Medicine, 339:2015-2017.

[2] Hollermann, S., Melznerand, H. \& Bargstadt, J. (2012). BIM - A challenge for communication between parties involved in construction. In Gudnason, G. \& Schere, R. (Eds) eWork and eBusiness in Architecture, Engineering and Construction (ECPPM). CRC press, 833-838.

[3] National Breast Cancer Centre, (2005). Multidisciplinary Meetings for Cancer Care: A Guide for Health Service Providers. National Breast Cancer Centre, Camperdown, Sydney.

[4] Canadian Medical Protective Association, (2011). Strengthening Inter-Professional Communication. http://www.cmpa-

acpm.ca/cmpapd04/docs/resourcefiles/perspective/2011/01/pdf/co m_p1101_4-e.pdf Accessed: 10/7/2012.

[5] Fox, L.A. (2008). The Impact of Inter-professional Communication/collaboration during times of Transition for Cancer Patients with Advanced Disease - Systematic Analysis Using an Electronic Learning Module, BScN Thesis, University of Victoria.

[6] Huang, G., Medlam, G., Lee, J., Billingsley, S., et al., (2005). Error in the Delivery of Radiation Therapy: Results of a Quality Assurance Review. International Journal of Radiation Oncology Biology Physics, 61(5):1590-1595.

[7] Royal College of Surgeons in England, (2007). Safe Handover: Guidance from the Working Time, Directive Working Party. Guidance on Safe Handover. London.

[8] Lee, S.M. (2003). A Review of Language and other Communication Barriers in Health Care, U.S. Department of Health and Human Services.

[9] World Health Organisation, (2008). Radiotherapy Risk Profile, Technical Manual, 4.

[10] http://www.who.int/patientsafety/activities/technical/radiother apy_risk_profile.pdfAccessed: 06/01/2012.

[11] O`Daniel, M. \& Rosenstein, A. H. (2008). Professional Communication and Team Collaboration. In Hughes, R.G. ed. Patient Safety and Quality: An Evidence-Based Handbook for Nurses, 1-14.

[12] Joint Commission on Accreditation of Healthcare Organisations, (2005). The joint commission guide to improving staff communication, Joint Commission Resources, 6. Ilese, J.S. ed

[13] Institute of Medicine, (1999). To Err is Human: Building a safer health system. http://www.iom.edu/ /media/Files/Report\%20Files/1999/ToErrisHuman/To\%20Err\%20is\%20Human\%201999\%20\%20re port $\% 20$ brief.pdf. Accessed: 27/09/2012

[14] Mental Health Commission, (2006). Multidisciplinary Team Working: From Theory to Discussion Paper. Mental Health Commission: Dublin. 
[15] Kurtz, S., Sliverman, J. \& Draper, J. (1998). Teaching and Learning Communication Skills in Medicine. Abingdon, Oxen. Radcliffe Medical Press.

[16] Butow, P. (2001). The importance of communication skills to effective cancer care and support. NSW Public Health Bulletin, 12(10):272.

[17] Horii, S. C. (1997). Primer on Computers and Information Technology. Part four: A Nontechnical Introduction to DICOM Radiographics,

17:1297-1309 http://radiographics.rsna.org/content/17/5/1297.full.pdf + html Accessed: 10/7/2012.

[18] Indrajit, I. K. (2007). Digital Imaging and Communications in Medicine: A Basic Review. Indian Journal of Radiology and Imaging, 17:5-7.

[19] Iezzoni, L. I., O'Day, B. L., Killeen, M. \& Harker, H. (2004). Communicating about health care: observations from persons who are deaf or hard of hearing. Annals of Internal Medicine, 140(5):356-62. 